\title{
16 Trans-Fettsäuren
}

(c) Springer-Verlag GmbH Deutschland, ein Teil von Springer Nature 2018

D. Mathias, Fit und gesund von 1 bis Hundert

https://doi.org/10.1007/978-3-662-56307-6_16

Nicht alle Fette sind für hohe Brattemperaturen von $130-180{ }^{\circ} \mathrm{C}$ geeignet. Wasser im Fett wie z. B. in der Butter verdampft bei $100^{\circ} \mathrm{C}$ und beginnt dann zu spritzen. Begleitstoffe aus dem Fruchtfleisch kalt gepresster Öle können sich beim Erhitzen über $150{ }^{\circ} \mathrm{C}$ verändern und einen unangenehmen Geruch oder Geschmack entwickeln. Gute Bratfette enthalten also wenig Wasser, sind frei von Geruchsund Geschmacksstoffen und haben einen hohen Rauchpunkt. Beispiele hierfür sind Butterschmalz, Palmfett oder raffiniertes Rapsöl.

Nahrungsstoffe mit einem reichlichen Gehalt an mehrfach ungesättigten Fettsäuren verlieren beim Braten durch Reaktionen mit Sauerstoff ihre wertvollen Eigenschaften, weil durch die Oxidation Doppelbindungen in Einfachbindungen übergehen. Der gesundheitliche Nutzen von rohem oder gekochtem Fisch ist deshalb höher als der von Fisch in gebratener oder frittierter Zubereitung ( $\downarrow$ Kap. 37 und $>$ Kap. 38).

Ein anderer Effekt hoher Brattemperaturen besteht darin, dass für Sekundenbruchteile die Doppelbindungen aufgehen und dann in geringem Umfang Umlagerungen von den natürlichen cis- zu den gesundheitsschädlichen trans-Fettsäuren stattfinden können. Die trans-Fettsäuren steigern die Spiegel von LDL-Cholesterin und senken das HDL-Cholesterin (Dietz und Scanlon 2012; Yanai et al. 2015). Sie erhöhen so das Risiko für koronare Herzerkrankungen mit den möglichen Folgen eines Herzinfarkts oder Schlaganfalls (Brouwer et al. 2013). Trans-Fettsäuren können ferner endotheliale Dysfunktionen induzieren ( $\$$ Kap. 58 ), sie sind am Entstehen der Insulinresistenz beteiligt ( $\triangleright$ Kap. 45 ) und sie fördern die viszerale Adipositas (Micha und Mozaffarian 2009). Ein zu hoher Konsum von Transfetten steigert folglich auch die Sterblichkeit (de Souza et al. 2015). Ebenfalls besteht ein Zusammenhang zwischen ihrem Verzehr und einem vermehrten Auftreten von Depressionen (SanchezVillegas et al. 2011; Ginter und Simko 2016). Die Aufnahme der trans-Fettsäuren sollte deshalb begrenzt werden. Maximal $1 \%$ der Nahrungsenergie, das sind etwa 2-3g pro Tag, gelten als unbedenklich. Enthalten sind sie hauptsächlich in fetthaltigen Backwaren, Chips, Pommes frites, Trockensuppen, Fertiggerichten, Süßwaren und den meisten Margarinesorten. Ihre Mengen sind zubereitungsabhängig. In Deutschland besteht keine Kennzeichnungspflicht für die Höhe des Transfettanteils in Lebensmitteln. Eine solche existiert bisher nur in wenigen Ländern. Durch Mikroorganismen entstehen im Pansen von Wiederkäuern auch auf natürliche Weise trans-Fettsäuren. Milch und Rinderfett sind hier etwa mit 3-5 \% des jeweiligen Gesamtfettgehalts belastet.
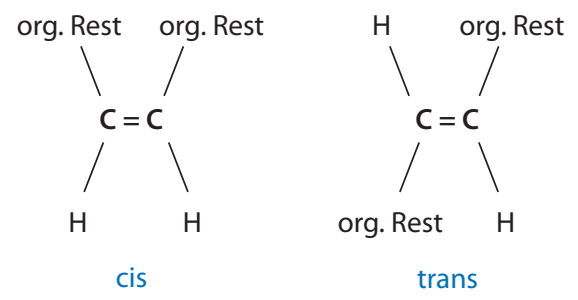

Abb. 16.1 cis-trans-Isomerie 